\title{
Threshold resummation beyond leading eikonal level
}

\section{Georges Grunberg*}

Centre de Physique Théorique, École Polytechnique,

91128 Palaiseau Cedex, France

E-mail: georges.grunberg@cpht.polytechnique.fr

\begin{abstract}
The modified evolution equation for parton distributions of Dokshitzer, Marchesini and Salam is extended to non-singlet Deep Inelastic Scattering coefficient functions and the physical evolution kernels which govern their scaling violation. Considering the $x \rightarrow 1$ limit, it is found that the leading next-to-eikonal logarithmic contributions to the momentum space physical kernels at any loop order can be expressed in term of the one loop cusp anomalous dimension, a result which can presumably be extended to all orders in $(1-x)$. Similar results hold for fragmentation functions in semi-inclusive $e^{+} e^{-}$annihilation. The method does not work for subleading next-to-eikonal logarithms, but, in the special case of the $F_{1}$ and $F_{T}$ structure and fragmentation functions, there are hints of the possible existence of an underlying Gribov-Lipatov like relation.
\end{abstract}

XVIII International Workshop on Deep-Inelastic Scattering and Related Subjects April 19 -23, 2010

Convitto della Calza, Firenze, Italy

${ }^{*}$ Speaker. 


\section{Threshold resummation in physical evolution kernels}

Consider a generic deep inelastic scattering (DIS) non-singlet structure function $\mathscr{F}\left(x, Q^{2}\right)=$ $\left\{2 F_{1}\left(x, Q^{2}\right), F_{2}\left(x, Q^{2}\right) / x\right\}$ at large $Q^{2}>>\Lambda^{2}$. We shall be interested in the elastic limit $x \rightarrow 1$ where the final state mass $W^{2} \sim(1-x) Q^{2}<<Q^{2}$. In this limit, large threshold $\ln (1-x)$ logarithms appear. Their resummation is by now standard [1,2], but usually performed in moment space. However, the result can also be expressed analytically in momentum space at the level of so-called "physical evolution kernels" which account for the physical scaling violation:

$$
\frac{\partial \mathscr{F}\left(x, Q^{2}\right)}{\partial \ln Q^{2}}=\int_{x}^{1} \frac{d z}{z} K\left(z, a_{s}\left(Q^{2}\right)\right) \mathscr{F}\left(x / z, Q^{2}\right) \equiv K \otimes \mathscr{F},
$$

where the "physical evolution kernel" $K\left(x, a_{s}\right)\left(a_{s}=\alpha_{s} / 4 \pi\right.$ is the $\overline{M S}$ coupling) embodies all the perturbative information about $\mathscr{F}$. For $x \rightarrow 1$ threshold resummation yields [3]:

$$
K\left(x, a_{s}\left(Q^{2}\right)\right) \sim\left[\frac{\mathscr{J}\left((1-x) Q^{2}\right)}{1-x}\right]_{+}+B_{\delta}^{D I S}\left(a_{s}\left(Q^{2}\right)\right) \delta(1-x),
$$

where $\mathscr{J}\left(Q^{2}\right)$ is a "physical anomalous dimension" (a renormalization scheme invariant quantity), related to the standard "cusp" $A\left(a_{s}\right)=\sum_{i=1}^{\infty} A_{i} a_{s}^{i}$ and final state "jet function" $B\left(a_{s}\right)=\sum_{i=1}^{\infty} B_{i} a_{s}^{i}$ anomalous dimensions by:

$$
\mathscr{J}\left(Q^{2}\right)=A\left(a_{s}\left(Q^{2}\right)\right)+\beta\left(a_{s}\left(Q^{2}\right)\right) \frac{d B\left(a_{s}\left(Q^{2}\right)\right)}{d a_{s}} \equiv \sum_{i=1}^{\infty} j_{i} a_{s}^{i}\left(Q^{2}\right) .
$$

The renormalization group invariance of $\mathscr{J}\left(Q^{2}\right)$ yields the standard relation:

$$
\begin{aligned}
\mathscr{J}\left((1-x) Q^{2}\right) & =j_{1} a_{s}+a_{s}^{2}\left[-j_{1} \beta_{0} L_{x}+j_{2}\right] \\
& +a_{s}^{3}\left[j_{1} \beta_{0}^{2} L_{x}^{2}-\left(j_{1} \beta_{1}+2 j_{2} \beta_{0}\right) L_{x}+j_{3}\right]+\mathscr{O}\left(a_{s}^{4}\right),
\end{aligned}
$$

where $L_{x} \equiv \ln (1-x)$ and $a_{s}=a_{s}\left(Q^{2}\right)$, from which the structure of all the eikonal logarithms in $K\left(x, a_{s}\left(Q^{2}\right)\right)$, which can be absorbed into the single scale $(1-x) Q^{2}$, can thus be derived.

However, no analogous result holds at the next-to-eikonal level (except [4] at large- $\beta_{0}$ ). Indeed, expanding

$$
K\left(x, a_{s}\right)=K_{0}(x) a_{s}+K_{1}(x) a_{s}^{2}+K_{2}(x) a_{s}^{3}+\mathscr{O}\left(a_{s}^{4}\right),
$$

the $K_{i}$ 's can be determined as combinations of splitting and coefficient functions. One gets:

$$
K_{0}(x)=P_{0}(x)=k_{10} p_{q q}(x)+\Delta_{1} \delta(1-x),
$$

with $k_{10}=A_{1}$ and $p_{q q}(x)=\frac{x}{1-x}+\frac{1}{2}(1-x)$. Moreover for $x \rightarrow 1$ one finds [5, 6], barring delta function contributions:

$$
\begin{aligned}
& K_{1}(x)=\frac{x}{1-x}\left(k_{21} L_{x}+k_{20}\right)+\left(h_{21} L_{x}+h_{20}\right)+\mathscr{O}\left((1-x) L_{x}\right) \\
& K_{2}(x)=\frac{x}{1-x}\left(k_{32} L_{x}^{2}+k_{31} L_{x}+k_{30}\right)+\left(h_{32} L_{x}^{2}+h_{31} L_{x}+h_{30}\right)+\mathscr{O}\left((1-x) L_{x}^{2}\right) .
\end{aligned}
$$


Despite the similar logarithmic structure, the next-to-eikonal logarithms $h_{i j}$ cannot [5] be obtained from a standard renormalization group resummation analogous to the one used (eq.(1.4)) for the eikonal logarithms $k_{i j}$.

\section{An alternative approach: the modified physical kernel}

Instead, consider [7] a modified physical evolution equation, similar to the one used in [8] (see also [9]) for parton distributions:

$$
\frac{\partial \mathscr{F}\left(x, Q^{2}\right)}{\partial \ln Q^{2}}=\int_{x}^{1} \frac{d z}{z} K\left(z, a_{s}\left(Q^{2}\right), \lambda\right) \mathscr{F}\left(x / z, Q^{2} / z^{\lambda}\right),
$$

where the arbitrary parameter $\lambda$ shall be set to 1 at the end. Expanding $\mathscr{F}\left(y, Q^{2} / z^{\lambda}\right)$ around $z=1$, one can relate $K\left(x, a_{s}, \lambda\right)$ to $K\left(x, a_{s}\right)$ :

$$
K\left(x, a_{s}, \lambda\right)=K\left(x, a_{s}\right)+\lambda\left[\ln x K\left(x, a_{s}, \lambda\right)\right] \otimes K\left(x, a_{s}\right)+\ldots .
$$

Solving perturbatively, one finds that for $x \rightarrow 1$ the corresponding expansion coefficients $K_{i}(x, \lambda)$ satisfy the analogue of eq.(1.7), with the same coefficients $k_{j i}$ 's of the eikonal logarithms, but with the coefficients of the leading next-to-eikonal logarithms given by:

$$
\begin{aligned}
& h_{21}(\lambda)=h_{21}-\lambda k_{10}^{2} \\
& h_{32}(\lambda)=h_{32}-\lambda \frac{3}{2} k_{21} k_{10} .
\end{aligned}
$$

Setting now $\lambda=1$, one observes that both $h_{21}(\lambda=1)$ and $h_{32}(\lambda=1)$ vanish, which means that $h_{21}=k_{10}^{2}=A_{1}^{2}=16 C_{F}^{2}$ and $h_{32}=\frac{3}{2} k_{21} k_{10}=-\frac{3}{2} \beta_{0} A_{1}^{2}=-24 \beta_{0} C_{F}^{2}$, which agree with the exact results in $[5,6]$. It should be stressed that, whereas $h_{21}$ is contributed by the two loop splitting function alone (and thus one simply recovers in this case the result of [8]), $h_{32}$ is instead contributed only by the one and two loop coefficient functions, which represents a new result. Similar results are obtained for the coefficients $h_{j i}(j=i+1)$ of the leading next-to-eikonal logarithms at any loop order, which can all be expressed in term of the one loop cusp anomalous dimension assuming the corresponding $h_{j i}(\lambda)$ vanish for $\lambda=1$. In particular, one predicts $h_{43}=\frac{4}{3} k_{10} k_{32}+\frac{1}{2} k_{21}^{2}=$ $\frac{11}{6} \beta_{0}^{2} A_{1}^{2}=\frac{88}{3} \beta_{0}^{2} C_{F}^{2}$, which is correct [5, 6], and $h_{54}=\frac{5}{4} k_{10} k_{43}+\frac{5}{6} k_{21} k_{32}=-\frac{25}{12} \beta_{0}^{3} A_{1}^{2}=-\frac{100}{3} \beta_{0}^{3} C_{F}^{2}$, which remains to be checked.

Similar results are obtained for the coefficients $f_{j i}(j=i+1)$ of the leading next-to-next-to eikonal logarithms, defined by:

$$
\left.K_{i}(x)\right|_{\mathrm{LL}}=L_{x}^{i}\left[p_{q q}(x) k_{j i}+h_{j i}+(1-x) f_{j i}+\mathscr{O}\left((1-x)^{2}\right)\right],
$$

where the full one loop prefactor $p_{q q}(x)$ should be used in the leading term to define the $f_{j i}$ 's. The corresponding $f_{j i}(\lambda)$ coefficients in $K_{i}(x, \lambda)$ are given by:

$$
f_{21}(\lambda)=f_{21}+\lambda \frac{1}{2} k_{10}^{2}
$$




$$
\begin{aligned}
& f_{32}(\lambda)=f_{32}-\lambda\left(-\frac{3}{4} k_{10} k_{21}+k_{10} h_{21}\right)+\lambda^{2} \frac{1}{2} k_{10}^{3} \\
& f_{43}(\lambda)=f_{43}-\lambda\left(-\frac{2}{3} k_{10} k_{32}+\frac{1}{2}\left(h_{21}-\frac{1}{2} k_{21}\right) k_{21}+k_{10} h_{32}\right)+\lambda^{2} k_{10}^{2} k_{21},
\end{aligned}
$$

where one notes the presence of contributions quadratic in $\lambda$. Assuming the $f_{j i}(\lambda)$ 's vanish for $\lambda=1$, the resulting predictions for the $f_{j i}$ 's $(j=i+1)$ are again found to agree with the exact results of [6].

\section{Fragmentation functions}

Similar results hold for physical evolution kernels associated to fragmentation functions in semi-inclusive $e^{+} e^{-}$annihilation (SIA), provided one sets $\lambda=-1$ in the modified evolution equation:

$$
\frac{\partial \mathscr{F}_{S I A}\left(x, Q^{2}\right)}{\partial \ln Q^{2}}=\int_{x}^{1} \frac{d z}{z} K_{S I A}\left(z, a_{s}\left(Q^{2}\right), \lambda\right) \mathscr{F}_{S I A}\left(x / z, Q^{2} / z^{\lambda}\right),
$$

where $\mathscr{F}_{S I A}=\left\{\mathscr{F}_{T}, \mathscr{F}_{T+L}\right\}$ denotes a generic non-singlet fragmentation function (I use the notation of [6]). At the leading eikonal level, threshold resummation [10] can be summarized in the standard SIA physical evolution kernel by:

$$
K_{S I A}\left(x, a_{s}\left(Q^{2}\right)\right) \sim\left[\frac{\mathscr{J}\left((1-x) Q^{2}\right)}{1-x}\right]_{+}+B_{\delta}^{S I A}\left(a_{s}\left(Q^{2}\right)\right) \delta(1-x),
$$

where the "physical anomalous dimension" $\mathscr{J}\left(Q^{2}\right)$ (hence the $k_{j i}$ 's) are the same for DIS and SIA, as follows from the results in [11]. Assuming the leading threshold logarithms vanish beyond the leading eikonal level in the modified SIA evolution kernel for $\lambda=-1$, and setting $\lambda=-1$ in eq. (2.3) and (2.5), one derives predictions for $h_{j i}^{S I A}$ and $f_{j i}^{S I A}(j=i+1)$ which again agree with the exact results of [6]. In particular, one finds that $h_{j i}^{S I A}=-h_{j i}$.

\section{Subleading next-to-eikonal logarithms}

The previous approach does not work for subleading next-to-eikonal logarithms, namely the latter do not vanish in the modified physical evolution kernels for $\lambda= \pm 1$. The following facts are nevertheless worth quoting:

- At large $\beta_{0}$, we have a generalization [4] of the leading eikonal single scale ansatz (which takes care of all subleading logarithms) to any eikonal order:

$$
\begin{aligned}
\left.K\left(x, Q^{2}\right)\right|_{\text {large } \beta_{0}}= & {\left[\left.\frac{x}{1-x} \mathscr{J}\left(W^{2}\right)\right|_{\text {large } \beta_{0}}\right]_{+}+(\delta(1-x) \text { term }) } \\
& +\left.\mathscr{J}_{0}\left(W^{2}\right)\right|_{\text {large } \beta_{0}}+\left.(1-x) \mathscr{J}_{1}\left(W^{2}\right)\right|_{\text {large } \beta_{0}}+\ldots
\end{aligned}
$$

where $W^{2}=(1-x) Q^{2}$, and the $\mathscr{J}_{i}$ 's (except the leading eikonal one) are structure function dependent . A similar result holds for $\left.K_{S I A}\left(x, Q^{2}\right)\right|_{\text {large } \beta_{0}}$. 
- There are remarkable relations between the momentum space next-to-leading threshold logarithms of the (DIS) $F_{1}$ and the corresponding (SIA) $F_{T}$ transverse fragmentation function physical evolution kernels at the next-to-eikonal level. Namely, using the moment space results of [6], one can derive the following momentum space relations:

1) At two loop for the $\mathscr{O}\left(L_{x}^{0}\right)$ next-to-eikonal constant term:

$$
\begin{aligned}
& h_{20}^{\left(F_{1}\right)}=\left.h_{20}^{\left(F_{1}\right)}\right|_{\text {large } \beta_{0}}+\Delta h_{20} \\
& h_{20}^{\left(F_{T}\right)}=\left.h_{20}^{\left(F_{T}\right)}\right|_{\text {large } \beta_{0}}-\Delta h_{20},
\end{aligned}
$$

with $\left.h_{20}^{\left(F_{1}\right)}\right|_{\text {large } \beta_{0}}=-11 \beta_{0} C_{F},\left.h_{20}^{\left(F_{T}\right)}\right|_{\text {large } \beta_{0}}=7 \beta_{0} C_{F}$, and $\Delta h_{20}=A_{1} \Delta_{1}=12 C_{F}^{2}$.

2) At three loop for the single $\mathscr{O}\left(L_{x}\right)$ next-to-eikonal logarithms:

$$
\begin{aligned}
& h_{31}^{\left(F_{1}\right)}=\left.h_{31}^{\left(F_{1}\right)}\right|_{\text {large } \beta_{0}}+\Delta h_{31} \\
& h_{31}^{\left(F_{T}\right)}=\left.h_{31}^{\left(F_{T}\right)}\right|_{\text {large } \beta_{0}}-\Delta h_{31},
\end{aligned}
$$

with $\left.h_{31}^{\left(F_{1}\right)}\right|_{\text {large } \beta_{0}}=-\left.2 \beta_{0} h_{20}^{\left(F_{1}\right)}\right|_{\text {large } \beta_{0}}=22 C_{F} \beta_{0}^{2},\left.h_{31}^{\left(F_{T}\right)}\right|_{\text {large } \beta_{0}}=-\left.2 \beta_{0} h_{20}^{\left(F_{T}\right)}\right|_{\text {large } \beta_{0}}=-14 C_{F} \beta_{0}^{2}$, and:

$$
\Delta h_{31}=2 A_{1} A_{2}-20 \beta_{0} C_{F} C_{A}+20 \beta_{0} C_{F}^{2}
$$

3) At four loop for the double $\mathscr{O}\left(L_{x}^{2}\right)$ next-to-eikonal logarithms:

$$
\begin{aligned}
& h_{42}^{\left(F_{1}\right)}=\left.h_{42}^{\left(F_{1}\right)}\right|_{\text {large } \beta_{0}}+\Delta h_{42} \\
& h_{42}^{\left(F_{T}\right)}=\left.h_{42}^{\left(F_{T}\right)}\right|_{\text {large } \beta_{0}}-\Delta h_{42},
\end{aligned}
$$

with $\left.h_{42}^{\left(F_{1}\right)}\right|_{\text {large } \beta_{0}}=\left.3 \beta_{0}^{2} h_{20}^{\left(F_{1}\right)}\right|_{\text {large } \beta_{0}}=-33 C_{F} \beta_{0}^{3},\left.h_{42}^{\left(F_{T}\right)}\right|_{\text {large } \beta_{0}}=\left.3 \beta_{0}^{2} h_{20}^{\left(F_{T}\right)}\right|_{\text {large } \beta_{0}}=21 C_{F} \beta_{0}^{3}$, and:

$\Delta h_{42}=-24 \beta_{1} C_{F}^{2}+45 \beta_{0}^{2} C_{F} C_{A}-178 \beta_{0}^{2} C_{F}^{2}-\left(47-10 \zeta_{2}\right) \beta_{0} C_{F} C_{A}^{2}-\left(60-140 \zeta_{2}\right) \beta_{0} C_{F}^{2} C_{A}-16 \beta_{0} C_{F}^{3}$.

The large- $\beta_{0}$ parts are consistent with eq.(4.1), while the remaining $\pm \Delta h_{i j}$ corrections are suggestive of an underlying (yet to be discovered) Gribov-Lipatov like relation [14]. 
- No such relations exist between the DIS $F_{2}$ structure function and the corresponding total angle-integrated $F_{T+L}$ fragmentation function. This fact suggests to focus instead on the momentum space physical evolution kernels of the longitudinal structure $[12,13]$ and fragmentation functions. Indeed, some observations in [6] do suggest that the $\mathscr{O}(1 /(1-x))$ part of the spacelike and timelike longitudinal evolution kernels might actually be identical to any logarithmic accuracy.

\section{Conclusions}

- Using a kinematically modified [8] physical evolution equation, evidence has been given that the leading threshold logarithms at any eikonal order in the momentum space DIS and SIA non-singlet physical evolution kernels can be expressed in term of the one loop cusp anomalous dimension $A_{1}$, which represents the first step towards threshold resummation beyond the leading eikonal level. This result also explains the observed universality $[5,6]$ of the leading logarithmic contributions to the physical kernels of the various non-singlet structure functions at any order [6] in $1-x$.

- The present approach does not work for subleading next-to-eikonal logarithms. However, there are hints of the possible existence of an underlying (yet to be understood) GribovLipatov like relation in the special case of the $F_{1}$ DIS structure function and the corresponding $F_{T}$ SIA transverse fragmentation function.

\section{References}

[1] G. Sterman, Nucl. Phys. B281 (1987) 310.

[2] S. Catani and L. Trentadue, Nucl. Phys. B327 (1989) 323.

[3] E. Gardi and G. Grunberg, Nucl. Phys. B 794 (2008) 61 [arXiv:0709.2877 [hep-ph]].

[4] G. Grunberg, arXiv:0710.5693 [hep-ph].

[5] G. Grunberg and V. Ravindran, JHEP 0910 (2009) 055 [arXiv:0902.2702 [hep-ph]].

[6] S. Moch and A. Vogt, JHEP 0911 (2009) 099 [arXiv:0909.2124 [hep-ph]].

[7] G. Grunberg, Phys. Lett. B 687 (2010) 405 [arXiv:0911.4471 [hep-ph]].

[8] Yu. L. Dokshitzer, G. Marchesini and G. P. Salam, Phys. Lett. B 634 (2006) 504 [arXiv:hep-ph/0511302].

[9] B. Basso and G. P. Korchemsky, Nucl. Phys. B 775 (2007) 1 [arXiv:hep-th/0612247].

[10] M. Cacciari and S. Catani, Nucl. Phys. B 617 (2001) 253 [arXiv:hep-ph/0107138].

[11] S. Moch and A. Vogt, Phys. Lett. B 680 (2009) 239 [arXiv:0908.2746 [hep-ph]].

[12] S. Moch and A. Vogt, JHEP 0904 (2009) 081 [arXiv:0902.2342 [hep-ph]].

[13] G. Grunberg, arXiv:0910.3894 [hep-ph].

[14] V. N. Gribov and L. N. Lipatov, Sov. J. Nucl. Phys. 15 (1972) 438, ibid. 15 (1972) 675. 